\title{
General Psychiatry Mental health facility codesign: A new research method for integrating the service user voice in design processes using virtual reality
}

To cite: Liddicoat S. Mental health facility codesign: A new research method for integrating the service user voice in design processes using virtual reality. General Psychiatry 2019;32:e100061. doi:10.1136/ gpsych-2019-100061

Received 17 February 2019 Revised 28 April 2019 Accepted 30 April 2019

\section{Check for updates}

(c) Author(s) (or their employer(s)) 2019. Re-use permitted under CC BY-NC. No commercial re-use. See rights and permissions. Published by BMJ.

${ }^{1}$ School of Design, Faculty of Health, Arts and Design, Swinburne University of Technology, Melbourne, Victoria, Australia

${ }^{2}$ Faculty of Architecture, Building and Planning, The University of Melbourne, Melbourne, Victoria, Australia

Correspondence to Dr Stephanie Liddicoat; stephanie.liddicoat@unimelb. edu.au

\section{ABSTRACT}

Background Within healthcare environments, the emerging field of evidence-based design (EBD) explores the links between wellbeing and good design practice of the built environment.

Aim By optimising both design processes and design outcomes, knowledge produced within this field seeks to improve staff performance, augment patient healing and enhance service outcomes and experiences.

Methods In a prior study by the author, a mental health service building design was developed which integrated feedback from mental health service users relative to what aspects of the built environments of their care would enhance their service outcomes and experiences, encourage them to avail themselves of services and/or engage in therapy, and those that would reduce their willingness to avail themselves of services.

Results The research project protocol detailed here is the final testing stage of this body of work, where service users are invited to evaluate the final building design, experienced through virtual reality. This study addresses a gap in the literature, and aims to advance the field of EBD, and codesign with mental health service users, using virtual reality.

Conclusions This research method details the aims, study design, methods and limitations of the study, with recommendations for future researchers.

\section{INTRODUCTION}

Environments providing mental health services are regarded within clinical literature as having an effect on a patient's sense of well-being. ${ }^{1}$ Patients' experience of such spaces can have a highly emotional dimension, ${ }^{2}$ which is suggestive that environment design should be investigated as a potential means to influence therapeutic efficacy. Further, individuals have differing abilities to censor or suppress their environments, ${ }^{3}$ and a stressed patient has reduced capacity to exclude environmental distractions, ${ }^{4}$ suggesting mental health service environments may have more impact for these individuals who often arrive in a distressed state.

Within healthcare environments, the emerging field of evidence-based design (EBD) explores the links between wellbeing and good design practice of the built environment. By optimizing both design processes and design outcomes, knowledge produced within this field seeks to improve staff performance, augment patient healing and enhance service outcomes and experiences. With recent developments in technology and advanced modelling systems, novel forms of architecture and representation are now possible, freed from the limitations of the orthogonal drawing system of plan, section and elevation, and two-dimensional representations such as photographs. ${ }^{5}$ The design of architecture is now being created through digital, three-dimensional drawing techniques and virtual reality (VR) experiences, which open up the possibilities for the inhabitation of architectural representations and propose new kinds of architectural spaces. Research focused on direct comparisons between real and virtual environments has investigated how cognitive and affective environmental appraisal and human patterns of movement correspond in both environments. ${ }^{67}$ VR can also support preoccupancy building evaluations by the proposed end users. However, only a handful of existing studies have investigated this potential. ${ }^{8}$ Experiential qualities of space can impact decision making, activities and spatial inhabitation. For example, studies have found that darker virtual environments feel more unpleasant, ${ }^{9}$ and participants tend to avoid darker corridors when presented with a choice of light-filled corridors. ${ }^{10}$ Therefore, 'it is important to not only look at behavioral outcomes, but to understand how people experience virtual spaces'. ${ }^{11}$

\section{THE PROPOSED STUDY}

This study represents a final evaluative step in an overall body of work developed by the author. In a prior study (study A), a mental 
health service building design was developed through an exploratory research project investigating the needs of service users of mental health services relative to the built environments delivering these services. This building is a community-based mental health facility providing therapy and counselling services for outpatients. Its design is suitable for such staff as mental health nurses, occupational therapists, psychologists and psychiatrists to provide community-based care. It includes a reception/ waiting area, a selection of consulting suites at a variety of sizes, one group therapy/multipurpose room, staff offices, two breakout kitchens/lounge areas, two courtyards and bathroom facilities as required. This building design integrated feedback from mental health service users relative to what aspects of the built environment would enhance their service outcomes and experiences, encourage them to avail themselves of services and/or engage in therapy, and those that would reduce their willingness to avail themselves of services. The research project detailed here (study B) is the final testing stage of this body of work, where service users are invited to evaluate the final building design, experienced through VR.

\section{Aims}

The aim of this study is to address the following investigative areas:

- Applicability of VR as a participatory design and research tool by testing its potential in an empirical study.

- Understanding the supportive and unsupportive aspects of the built environment which might influence mental health service user outcomes and experiences.

- Efficacy of EBD in capturing mental health service user voices regarding the design of built therapeutic environments.

Thus, the research questions formed were the following:

- What are the opportunities and limitations afforded by VR as a codesign methodology with mental health service users?

- What are the spatial or built environment perceptions which are influential in creating supportive mental health service experiences?

- How can architectural design contribute to improved therapeutic experiences for mental health service users?

A mixed methods approach will be used to investigate these questions, working primarily with qualitative data from interviews and some quantitative data through VR device data collection.

\section{Design}

Itemised methods are as follows:

- Testing the VR model as an iterative codesign research tool. Researchers learn how to deploy VR and to facilitate the set-up of the model. The activities take place in the VR Lab at the University of Melbourne and will only involve the participation of members of the research team. The lab testing will inform the deployment of the VR model in the interview stage of the project.

- A participatory research approach will be employed in the data collection, analysis and collation of findings of this research using the Centre for eHealth and Wellbeing Research Roadmap to help plan, coordinate and execute the participatory development process; ${ }^{12}{ }^{13}$ this methodology captures input and data from researchers and mental health service users collectively to engage in processes of enquiry, where parties 'become partners in research instead of only consumers of it'. ${ }^{14}$

- Recruitment of mental health service users to interview with the assistance of two mental health services based in a large Australian city.

- Mental health service users experience the designed environment in VR and participate in a post-VR interview. A trained mental health professional, such as a case manager, will be present during the interview.

- Analysis and dissemination of results.

A matrix of findings from study A was established to inform the development of interview questions/framework to be used in study B (see table 1). Detection instruments and specific questions were devised and refined based on each of the factors affecting outcomes and experiences, together with the spaces of care (settings).

\section{Setting}

Two mental health services based in a large Australian city will support the recruitment and selection so that (1) participants deemed unsuitable will not be unknowingly recruited, and (2) risk to participants will be minimised in regard to confidential health information being accessed by researchers. For example, service users who are acutely psychotically unwell will not be suitable candidates. However, we acknowledge that it is important that more vulnerable individuals are not arbitrarily excluded from participation in research, and that even symptomatic individuals can give valid and valuable input to the research investigation. These services will make the initial approach to users of its services based on their knowledge of candidates and their history of illnesses and treatments.

\section{Target population and recruitment}

The study aims to recruit a minimum of 15 participants (adult individuals) who are/have been using mental health services for voluntary participation in a VR walkthrough and a follow-up semistructured interview.

The two mental health services will make the approach to potential participants and provide them information about the study as per a prescribed email invitation. On giving their consent to contact, a member of the research team will contact the service user to provide each individual participant with a plain language statement and consent form. Once written consent is received, the researcher will contact participants to make specific appointments for interviews. Participant names will be 
Table 1 Relations between built environment and issues that influence the service user outcomes and experiences: matrix of findings from prior study

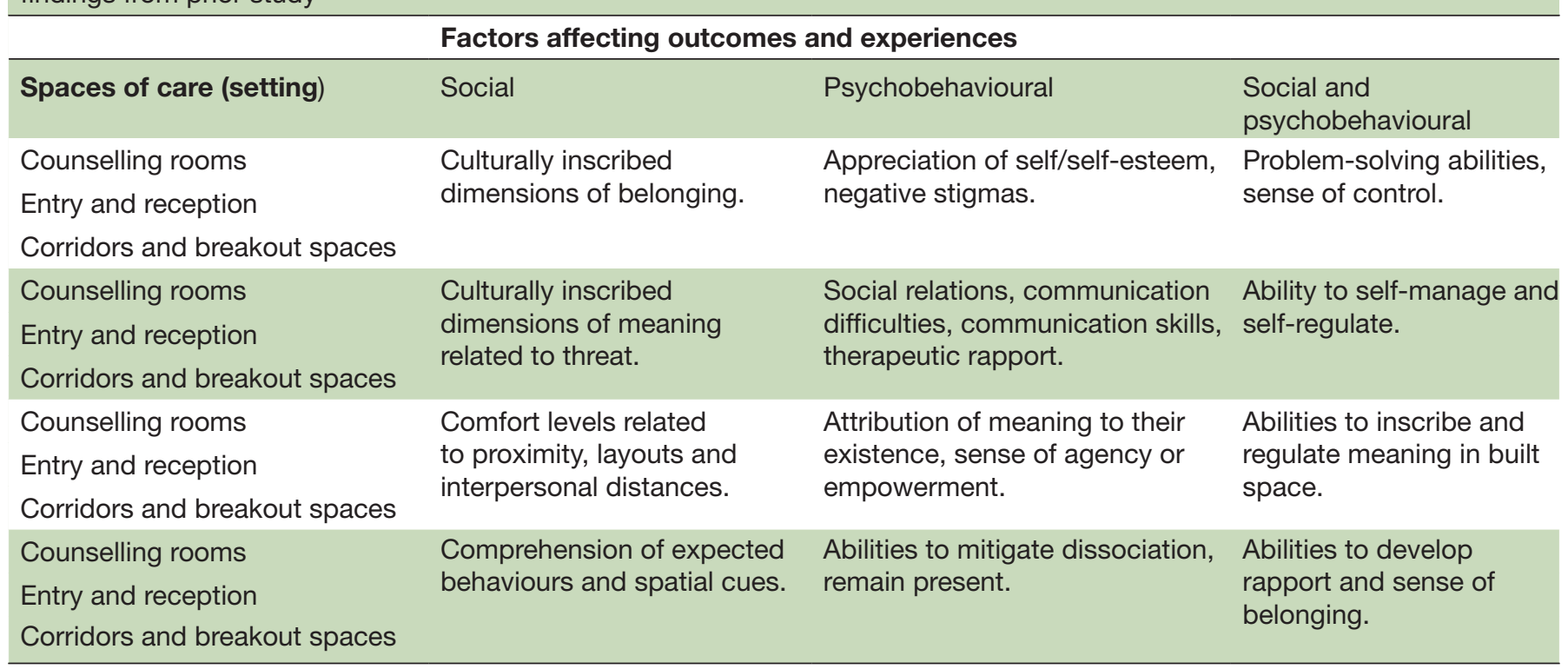

anonymised and/or participants assigned pseudonyms so that anonymity will be protected. Analysis of interview transcripts will use pseudonyms (eg, 'Participant 01') to refer to each transcript, to minimise risk of linking participants to identifiable data.

\section{Sample size}

The study aims to recruit a minimum of 15 participants who are/have been mental health service users. They will be recruited for voluntary participation in a VR walkthrough and a follow-up semistructured interview. Being a relatively new area of research, we base these participant numbers on recent studies ${ }^{15-18}$ and suitable sample sizes for indepth qualitative research. ${ }^{19}$

\section{Procedures and materials}

Each interviewee will be provided with the list of intended interview questions prior, and their consent will be sought to the audio recording of the interview. Each VR walkthrough and interview will last approximately 30-45 min (up to $60 \mathrm{~min}$ ) for both activities.

\section{The VR walk-through}

The VR walk-through is a model of an architectural building (produced through codesign with service users of mental health services during study A). This VR walkthrough is not intended to act as a therapeutic environment as part of a mental health intervention/treatment. Rather, it is a prototype building design. The purpose of the VR walk-through is to enable the research team to better understand participant preferences in terms of their built environments, using VR as the medium to show them the proposed building design (as opposed to other commonly used methods, such as photographs) and gain their feedback. The VR walk-through model involves a building which is not inhabited in VR by any other individual (real or virtual).

\section{The interviews}

Given the interplay between architectural design and quality of care, several researchers underscore the need for cooperation among architects/designers and the staff and service users who will work and experience the facilities they create. $^{20-22}$ Approaches which prioritise user experience, draw from interdisciplinary knowledge, use codesign methods, and include provision for prototyping, testing and refinement of design solutions are emphasised as key to advancing the EBD field. ${ }^{23}{ }^{24}$ Several studies underscore the importance of effectively translating research findings into concepts that are accessible and useable to design practice. ${ }^{25} 26$ EBD integrates the cyclical nature of design and research, with the common goal of continuously re-evaluating design decisions and improving design solutions. It is suggested that studies within the EBD field should measure the implementation of the client's guiding principles and specific user needs. ${ }^{25}$ Preoccupancy and postoccupancy studies typically involve survey and interview designs to gain feedback from users on features such as light, noise, aesthetics, safety, privacy and various dimensions of experiences, as determined by the guiding principles of the design and the relevant research literature. $^{27}$

However, such an approach is rarely carried out in full; the final step, of evaluating the efficacy of design solutions/guidelines, is often omitted. The guidelines currently available to assist the designers of mental health environments specifically have not been prototyped or tested. $^{28}$ This situation is reflected in architectural practice, where postoccupancy evaluations of healthcare projects are rare, as the funding is often expended in the construction phase (personal correspondence with Melbourne-based healthcare architects: Lyons, Billard Leece Partnership and Hobart-based healthcare architects X-Squared). Using VR offers significant potentials 


\begin{tabular}{|c|c|}
\hline Environment & Experience \\
\hline $\begin{array}{l}\text { Focusing on the detailed } \\
\text { environmental aspects of } \\
\text { spaces or places and their } \\
\text { impact on quality of care, } \\
\text { and delivery of care }\end{array}$ & $\begin{array}{c}\text { Focusing on design's } \\
\text { impact on service users } \\
\text { and their satisfaction with } \\
\text { care }\end{array}$ \\
\hline \multirow[t]{5}{*}{$\begin{array}{c}\text { Spaces: Reception, } \\
\text { Waiting, Consulting suites, } \\
\text { Break-out areas, } \\
\text { Courtyards, Corridor } \\
\text { spaces }\end{array}$} & $\begin{array}{l}\text { Impact of intuitive } \\
\text { dimensions of wayfinding } \\
\text { on patient satisfaction } \\
\text { Use of positive distractions } \\
\text { (such as nature) on the } \\
\text { care experience }\end{array}$ \\
\hline & $\begin{array}{l}\text { Use of material palette in } \\
\text { moderating the care } \\
\text { experience }\end{array}$ \\
\hline & $\begin{array}{l}\text { Dimensions of light levels } \\
\text { and perceived comfort }\end{array}$ \\
\hline & $\begin{array}{l}\text { Dimensions of personal } \\
\text { space, scale and territories }\end{array}$ \\
\hline & $\begin{array}{l}\text { Dimensions related to } \\
\text { furniture, objects and } \\
\text { other props as related to } \\
\text { perceived care experiences }\end{array}$ \\
\hline
\end{tabular}

Figure 1 Core areas of evaluation.

to advance the EBD field, by providing a time-effective and cost-effective means to gather a broad range of stakeholder feedback on building designs.

Interview questions developed for this study will broadly cover attitudes to supportive and unsupportive aspects of the built environment which might influence their service use outcomes and experiences. Interview questions cover the core areas of evaluation for this project (see figure 1), determined by the findings from study A and relevant research literature. VR as a tool to obtain participant feedback on building designs has also been piloted by the author; empirical research was conducted related to analysis of a built environment in VR, and this process has assisted the author to define and inform the interview questions to be used in study B.

During the semistructured interviews, the researcher will use the thematic framework, developed through the preceding project, in order to seek clarification from participants as required (see figure 2). Together, this process will assist in the identification and evaluation of the supportive and non-supportive aspects of the building design, as experienced by the participants. Through

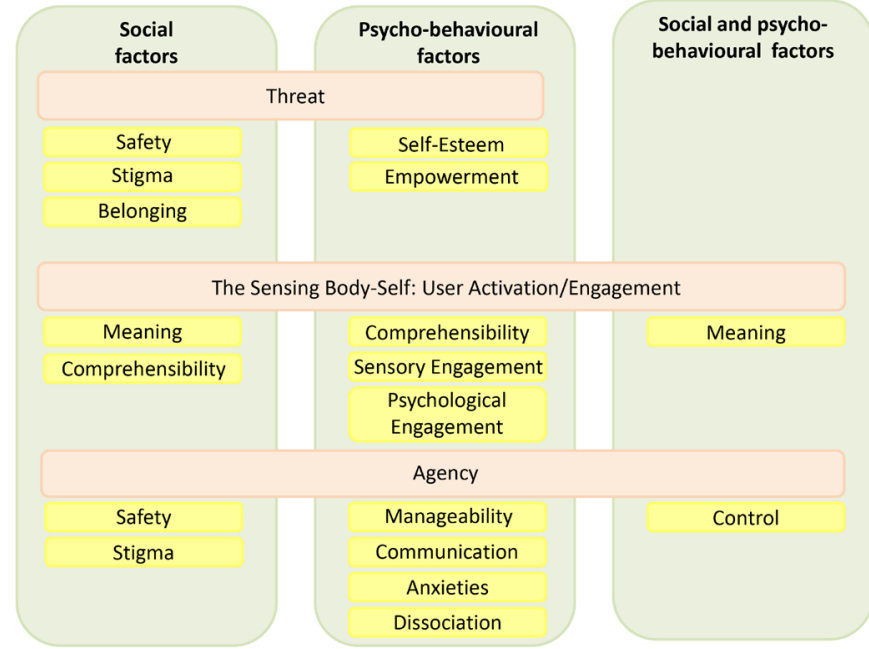

Figure 2 Thematic framework.

these interviews, the study will examine user perceptions in order to identify possible architectural interventions which may be supportive within mental health service facility design, and the various elements of the design that are important or significant to them in supporting their service outcomes and experiences.

\section{Screening}

The following are the inclusion criteria for the selection of study participants:

- Adult users of a mental health service.

- Have used a mental health service for over 1 year.

The following are the exclusion criteria:

- Young adults and children under 18 years of age.

- Users who are diagnosed with any mental health issue which may endanger themselves and/or members of the research team.

- Users who may not be fluent in the English language.

- Users who have a pre-existing illness/condition which may be exacerbated by virtual environments, such as sensitivity to bright lights from screens (mental health service to provide assistance).

\section{Data analysis}

The interview data will primarily be analysed through a thematic network analysis (using NVivo, Saturate or similar software tools). The lead researcher will examine the data to identify recurring themes that relate to the key research questions. These themes will be discussed and refined with the other project researchers. The analysis will broadly follow the thematic analysis methods outlined by Attride-Stirling. ${ }^{29}$

\section{DISCUSSION}

Anticipated benefits are that the project will result in (1) a toolkit set of guidance principles on access, design, community engagement, well-being measures and VR interventions, which could be used by mental health services/stakeholders; (2) published analyses of the design and role of therapeutic VR environments to 
support health self-management and its impact on service user experiences of mental health services; (3) publications analysing the role of VR in research with mental health service users; and (4) published access and design guidance to enhance codesign processes with mental health service users and communities.

\section{STRENGTHS AND LIMITATIONS}

The study reported here has two key limitations. The first is that it does not take steps to calibrate or compare participant responses to the virtual environments with any corresponding physical environments. A validation process is required in order that researchers can more explicitly comprehend the extent to which real and virtual spatial perceptions differ, and thus more accurately determine the reliability of these findings in informing real-world design practice. ${ }^{30}$ A second limitation is the small sample size, which prevents the identification of any trends regarding whether the characteristics of age, gender or cultural background influenced the perception of these architectural spaces.

\section{CONCLUSIONS}

The next phase for this study involves further testing of the VR environment included herein with patient cohorts within a mental health service waiting room. This would enable an exploration of how service users who are facing the emotions associated with a care appointment, such as frustration, boredom and anxiety, respond to the the virtual environments. These data could be further augmented with the incorporation of physiological measures, such as heart rate, blood pressure and cortisol measurements, alongside eye-tracking to pinpoint which elements within the environment are observed and hold attention. For a more holistic understanding of how healthcare settings affect service user outcomes and experiences, the inclusion of VR testing within a mixed methods research programme is advocated; inclusion of interviews, focus groups, observations, and participatory codesign workshops would provide a more comprehensive understanding of participant responses. ${ }^{30}$

Acknowledgements The author expresses thanks to Ann Borda for her suggestion to share this knowledge through a published protocol, and to Stephen Yuen for his work modelling the virtual reality experience.

Funding The authors have not declared a specific grant for this research from any funding agency in the public, commercial or not-for-profit sectors.

Competing interests None declared.

Patient consent for publication Not required.

Ethics approval Project title: Utilising virtual reality to enable codesign of therapeutic environments in mental health services. Ethics ID: 1853077.1, approved by the Humanities Law \& Social Sciences Human Ethics Sub-Committee at the University of Melbourne.

Provenance and peer review Not commissioned; externally peer reviewed. Data availability statement № additional data are available.

Open access This is an open access article distributed in accordance with the Creative Commons Attribution Non Commercial (CC BY-NC 4.0) license, which permits others to distribute, remix, adapt, build upon this work non-commercially, and license their derivative works on different terms, provided the original work is properly cited, appropriate credit is given, any changes made indicated, and the use is non-commercial. See: http://creativecommons.org/licenses/by-nc/4.0/.

\section{REFERENCES}

1 Gross R, Sasson Y, Zarhy M, et al. Healing environment in psychiatric Hospital design. Gen Hosp Psychiatry 1998;20:108-14.

2 Pressly PK, Heesacker M. The physical environment and counseling: a review of theory and research. Journal of Counselling and Development 2001;79:148-60.

3 Dijkstra K, Pieterse ME, Pruyn ATH. Individual differences in reactions towards color in simulated healthcare environments: the role of stimulus screening ability. J Environ Psychol 2008;28:268-77.

4 Samuelson DJ, Lindauer MS. Perception, evaluation, and performance in a neat and messy room by high and low sensation seekers. Environ Behav 1976;8:291-306.

5 Spurr S. Drawing the body in architecture. Architectural Theory Review 2009;14:322-32.

6 Westerdahl B, Suneson K, Wernemyr C, et al. Users' evaluation of a virtual reality architectural model compared with the experience of the completed building. Autom Constr 2006;15:150-65.

7 Comparing human wayfinding behavior in real and virtual environment. Proceedings of the 7th International Space Syntax Symposium, Stockholm, 2009.

8 Virtual environments for the evaluation of human performance: towards virtual occupancy evaluation in designed environments (VOE). Proceedings of the 11th International Conference on Computer Aided Architectural Design Research, Kumamoto, Japan, 2006.

9 Toet A, van Welie M, Houtkamp J. Is a dark virtual environment scary? Cyberpsychol Behav 2009;12:363-71.

10 What space SYNTAX does not know: movement triggers beyond integration. Proceedings of the 9th International Space Syntax Symposium, Seoul, South Korea, 2013.

11 Kuliga SF, Thrash T, Dalton RC, et al. Virtual reality as an empirical research tool - exploring user experience in a real building and a corresponding virtual model. Comput Environ Urban Syst 2015;54:363-75.

12 van Gemert-Pijnen JEWC, Nijland N, van Limburg M, et al. A holistic framework to improve the uptake and impact of eHealth technologies. J Med Internet Res 2011;13:e111.

13 Center for eHealth and Wellbeing Research (CeHRes). CeHRes RoadmapNot dated. Available: https://www.utwente.nl/en/bms/ ehealth/cehres/

14 Uggerhoj L. What is Practice Research in Social Work - Definitions, Barriers and Possibilities. Social Work \& Society 2011;9:45-59.

15 Wang E, Caldwell B. An empirical study of usability testing: heuristic evaluation vs. user testing. Proc Hum Factors Ergon Soc Annu Meet 2002;46:774-8

$16 \mathrm{Sim}$ J, Lewis M. The size of a pilot study for a clinical trial should be calculated in relation to considerations of precision and efficiency. $J$ Clin Epidemiol 2012;65:301-8.

17 Eichenberg C, Wolters C. Virtual realities in the treatment of mental disorders: A review of the current state of research. In: Virtual reality in psychological, medical and pedagogical applications. London: InTech, 2012: 35-64.

18 Maples-Keller JL, Bunnell BE, Kim S-J, et al. The use of virtual reality technology in the treatment of anxiety and other psychiatric disorders. Harv Rev Psychiatry 2017;25:103-13.

19 Nastasi B. Qualitative research: sampling and sample size considerations, 2014. Available: https://www.google.co.nz/url?sa= t\&rct=j\&q=\&esrc=s\&source $=$ web\&cd $=6 \& c a d=r j a \& u a c t=8 \& v e d=$ 0CFEQFjAF\&url=https\%3A\%2F\%2Fmy.laureate.net\%2FFaculty\% 2Fdocs\%2FFaculty\%2520Documents\%2Fqualit_res_smpl_size consid.doc\&ei=PTxHU43tDMeskgXnpoC4Cg\&usg=AFQjCNESrfQB olpITolsph6ZPpkE3rwnSg

20 Dvoskin JA, Radomski SJ, Bennett C, et al. Architectural design of a secure forensic state psychiatric hospital. Behav Sci Law 2002;20:481-93.

21 Howard T. Physical environment and use of space. In: Campling P, Davies S, Farquharson G, eds. From toxic institutions to therapeutic environments. London: Gaskell, 2004: 69-78.

22 Shepley MM, Watson A, Pitts F, et al. Mental and behavioral health settings: Importance \& effectiveness of environmental qualities \& features as perceived by staff. J Environ Psychol 2017;50:37-50.

23 Nakarada-Kordic I, Hayes N, Reay SD, et al. Co-designing for mental health: creative methods to engage young people experiencing psychosis. Design Health 2017;1:229-44. 
24 Bate P, Robert G. Experience-based design: from redesigning the system around the patient to co-designing services with the patient. Qual Saf Health Care 2006;15:307-10.

25 Fay L, Carll-White A, Harrell J. Coming full cycle: linking POE findings to design application. Health Environments Research \& Design Journal 2017;10:83-98.

26 Haq S, Pati D. The research-design interaction: lessons learned from an evidence-based design Studio. HERD 2010;3:75-92.

27 Kotzer AM, Zacharakis SK, Raynolds M, et al. Evaluation of the built environment: staff and family satisfaction pre- and post-occupancy of the children's Hospital. HERD 2011;4:60-78.
28 Shepley MM, Pasha S, Ferguson P, et al. Design research and mental and behavioural health facilities. The Center for Health Design: Concord, CA, 2013.

29 Attride-Stirling J. Thematic networks: an analytic tool for qualitative research. Qualitative Research 2001;1:385-405.

30 McLaughlan R, Liddicoat S. Evidence and affect: Employing virtual reality to probe what's missing from evidence-based design research. Design for Health 2019.

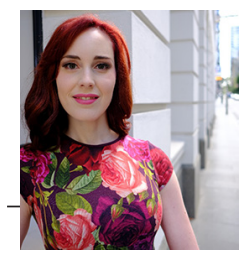

Dr. Stephanie Liddicoat completed a Master of Architecture (Professional) at Victoria University in Wellington, New Zealand, and has been working at the Faculty of Architecture, Building and Planning, University of Melbourne since 2013, and completed the PhD from the University of Melbourne, Australia (Faculty of Architecture, Building and Planning). She is engaged as a Research Fellow on the Australian Research Council Linkage project "Designing for Wellbeing: Realising the Benefits for Patients through Best Practice Hospital Design." In 2019 she was also appointed as a Lecturer in Architecture at the School of Design; Faculty of Health, Arts and Design at Swinburne University of Technology, Melbourne. Dr Liddicoat's research interests are at the nexus of architecture and health, and include how the built environment can support wellbeing within hospital settings, and the role of design practice in mental health service environments. She is also interested in participatory research methodologies, and furthering the field of evidence based design, through research and community engagement projects. Dr Liddicoat utilises emerging digital design and visualisation technologies in her research. Key to this is the recognition of how emerging technologies such as virtual reality, gaming, prototyping and mass customisation will impact not just design but also research processes (particularly participatory research processes). 